-at least a rudimentary acquaintance with what industry is all about". The latest figures of graduates trained per year (scientists and engineers combined) are roughly United Kingdom, 13,000 ; United States, 65,000 ; U.S.S.R., 130,000. One-third of Russian graduates in engineering are women. "It is one of our major follies that we do not in reality regard women as suitable for scientific careers. We thus divide our pool of potential talent by two."

"We are left with a population twice as large as we can grow food for, so that we are always going to be au fond more anxious than France or Sweden, and with very little in the way of natural resourcesby the standard of the great world powers, with nothing. The only real assets we have, in fact, are our wits. Those have served us pretty well, in two ways. We have a good deal of cunning, native or acquired, in the arts of getting on among ourselves : that is a strength, and we have been inventive and creative, possibly out of proportion to our numbers. Given these two assets, and they are our only ones, it should have been for us to understand the scientific revolution first, to educate ourselves to the limit and give a lead. In some fields, like atomic energy, we have done better than anyone could have predicted. Within the pattern, the rigid and erystallized pattern of our education and of the two cultures, we have been trying moderately hard to adjust ourselves. The historical warnings are all there. For instance, the Venetian Republic in its last half-century was guided by patriot men, who had immense political skill, who knew that the current of history had begun to flow against them. They were fond of the comfortable pattern of their life, just as we are fond of ours. They never found the will to break it."

There is yet another danger. The large masses of poor in the undeveloped countries will not allow themselves to live for ever in a world in which large sections have become rich through industry. A new missionary spirit, both human and technical, is essential.

"Closing the gap between our cultures is a necessity in the most abstract intellectual sense, as well as in the most practical. When those two senses have grown apart, then no society is going to be able to think with wisdom. For the sake of the intellectual life, for the sake of this country's special danger, for the sake of the western society living precariously rich among the poor, for the sake of the poor who need not be poor if there is intelligence in the world, it is obligatory for us and the Americans and the whole West to look at our education with fresh eyes. This is one of the cases where we and the Americans have the most to learn from each other. We have each a good deal to learn from the Russians, if we are not too proud. Incidentally, the Russians have a good deal to learn from us too." W. L. StMrNer

\title{
COAL SCIENCE
}

$\mathrm{T}$ HE third biennial International Conference on Coal Science was held at Valkenburg, in the Netherlands, during April 27-30. The Municipality kindly allowed the Conference to be held in the Municipal Theatre, and the greatest hospitality and interest were shown throughout by the burgomaster, F. A. A. H. Breekpot.

On this occasion the number of participating countries increased to fourteen, newcomers being East Germany, Czechoslovakia, Australia and India. Authors submitting papers were required to complete a form indicating, in telegraphic style, their main new conclusions and results, the methods and observations from which these were deduced, and any special limitations or assumptions involved in their interpretation. This information was found by the organizers to be more helpful than the conventional summary (or on occasions the paper itself) in deciding whether a paper was acceptable; it was also useful in evaluating the conclusions of the Conference and the interrelation of papers. The proceedings of this Conference will not be published as a whole ; but papers will be submitted by their authors to journals of their own choosing.

Discussion was unusually lively and fruitful, and a large proportion of novel work was presented. In several cases widely accepted ideas were apparently undermined, though it would be premature to assess the importance of the new evidence. For example, a suggestion arose, from the work of $\mathbf{S}$. Ergun and I. Wender on the $\mathrm{X}$-ray scattering of vitrinites reduced with lithium in ethylene diamine, that partially reduced aromatic and/or alicyclic molecules may lead to reflexions in the angular region where the (10) and (11) reflexions of aromatic mole- cules occur. This awaits direct experimental test, but if correct it may throw doubt upon the derivation of aromatic layer sizes in coal, for example, by Hirsch and Diamond. Ergun and Wender also demonstrated an increase of layer-spacing on reduction, even in graphite.

Similarly, an interpretation of data obtained on coal by high-resolution magnetic resonance spectroscopy, by J. K. Brown, W. R. Ladner and N. Sheppard, yielded a structural distribution of nonaromatic earbon atoms which may prove incompatible with the present consensus of opinion on the chemical structure of coal. As a third example, R. L. Bond and D. H. T. Spencer illustrated the inadequacy of existing interpretations of sorption data obtained with inert gases on coal and of heats of wetting of coal in polar liquids; a question thought by many to have been settled, at least in principle.

These, and the other thirty-three papers presented, were grouped under four heads, as follows.

\section{Chemical Reactions of Coal}

E. S. Hammack, H. G. Davis and F. B. Brown estimated the content of phenolic hydroxyl in vitrains by titration with sodium aminoethoxide in ethylene diamine, by trimethylsylylation and by acetvlation. They found reasonable agreement between these methods and noted the importance of particle size. Acetylation was studied by S. Delavareme, A. Halleux and H. Tschamler, who also confirmed the presence of quinone groups in coal extracts, by reductive acetylation and reduction with copper/ hydrogen sulphide. They observed the corresponding changes in the infra-red spectra and showed that the 
reduction was to a large extent reversible. In both papers, tests of the quantitative accuracy of the methods by experiments on model compounds such as quinones were reported. Fvidence for the presence of thioether groups in coals to the extent of 15--90 per cent of the organic sulphur content was put forward by L. Wnekowska. The difficulty of combining all known observations on the chemical and physical nature of coal, together with the elementary analysis, in a structural model was emphasized by P. H. Given, who by comparison of data and experiments with atomic models had been able to find only one satisfactory pattern of structure : the essential feature of this was that any pair of aromatic fused ring clusters is bound together by two methylene bridges.

E. J. Greenhow and J. W. Smith showed that pitch can be regarded as a solution of phenolic or basic compounds of medium molecular weight in a relatively non-polar solvent; results suggested that the physical properties were influenced by intermolecular association.

There were three papers on chromatographic separation of oxidation products of coal : by $\mathbf{F}$. Micheel, J. E. Germain and F. Valadon, and G. J. Lawson and S. G. Ward. The first author had isolated small yields of aniline, anthracene, substituted anthraquinone, tetraphene and fluoranthene from air- or nitric acid-oxidized coals; the second group had identified various benzene polycarboxylic acids, and the third, malonic, succinic and $l$-tartaric acids. S. Landa had studied hydrogenation of model substances, montan wax and humic acids extracted from brown-coal, with tungsten and molybdenum sulphide catalysts. Hydrocarbons with two to three fused aromatic rings and side-chains were found among the products from treatment of the humic acids.

\section{Ultra-fine Structure of Coals and Chars}

M. Wȩclewska discussed the influence of sorption on coals of organic vapours on their elasticity, and the consequent dimensional changes. S. Ergun, W. F. Donaldson and I. Breger showed that apparent changes in rank induced in coals by $\alpha$-particles originating from natural impregnation by uranium differed from those caused by normal coalification processes.

J. L. Soulé and S. Durif demonstrated, by measurements of small-angle X-ray scattering, the presence in a wide range of coals of pores of sizes $22-25 \mathrm{~A}$. and the variation of this characteristic with temperature of carbonization; they also deduced absolute values of specific internal surface. In the carbonization temperature range $500-700^{\circ} \mathrm{C}$. these surface areas agreed with those estimated by P. Chiche and $\mathrm{S}$. Prégermain from adsorption of water and methanol vapours.

Sorption of pyridine by carbonization products, their solubility in it and their swelling in various organic liquids, were studied by A. Ladam and P. Payen. Important changes were indicated in the region $400-500^{\circ}$ C. S. J. Gregg and M. I. Pope had studied the effect, on the electrical conductivity of coal artefacts, of pressure and of exposure to atmospheres of various humidities. J. J. Kipling and R. B. Wilson, from a study of the sorption of gases and vapours on chars prepared by carbonizing synthetic polymers, demonstrated the presence of microcapillaries of 5-10 A. diameter, molecular sieve properties of the structure, and the effect of steam activation in opening up this structure. A. Cameron and W. O. Stacey combined measurements of internal surface area, apparent density and measurement of internal volume with the porosimeter to demonstrate the presence of two distinct pore systems in chars prepared from brown-coals. The presence in 600$900^{\circ} \mathrm{C}$. chars of cavities of some $25 \mathrm{~A}$. linked by passages less than $10 \mathrm{~A}$. wide was inferred.

\section{Spectroscopy of Coals}

There were two papers on mass spectroscopy. One, by J. C. Robb and H. W. Holden, was concerned with the volatile products either volatilized or liberated by pyrolysis when coal was heated in the ion source. Interesting identifications in relation to temperature range were made. The other, by $R$. I. Reed and W. Snedden, was chiefly concerned with the fragments produced from aromatic compounds and thus widened the background available for interpreting results with coals. Electron magnetic resonance spectroscopy had been used to study coals in course of carbonization under vacuum, by J. Smidt and D. W. van Krevelen. In addition, the time of relaxation $T_{1}$ was measured as a function of tempera. ture. J. Uebersfeld had applied combined nuclear and electron magnetic resonance measurements and found evidence of interaction between protons and free radicals in coals of low rank, exchange interaction in those of high rank. Light spectroscopy was represented in two pepers, by R. A. Durie and J. Szewczyk and by S. Ergun and J. T. MacCartney. The first was concerned with a long wave-length absorption edge which moves into the infra-red with increasing temperature of pyrolysis. The magnitude of a corresponding energy gap in the structure was discussed in relation to the graph of polynuclear aromatic structures. In the other paper an attempt was made to establish specific reflectance of coals as a significant structural parameter, related to the hydrogen/carbon ratio and to the diamond and graphite structures as extremes.

\section{Carbonization}

C. Kröger, R. Brücker, M. Klatt and E. Bade had studied the maceral species exinite and vitrinite by pyrolysis under high vacuum. They discussed the origin of the liquid and gaseous products determined. A. M. Wandless and G. W. Fenton described coking tests, on coals of 84,85 and 87 per cent carbon content, that indicated a beneficial effect on the coke strength of the maceral inertinite as a constituent in the coal blend at the two lower levels of rank. D. W. van Krevelen, F. J. Huntjens and A. H. Wilms presented a thorough study of strength of coke in relation to particle size of coal carbonized. A. D. Dainton, W. G. Kaye and J. W. Phillips described the effect of macerals on volume changes in briquettes during carbonization. A. F. Boyer and P. Payen threw doubt on the interpretation of an apparent exothermic peak found in the differential thermal analysis of certain coals; they considered this to result from an increase of conductivity due to agglutination. Finally in this series there was an outstanding paper by P. M. J. Wolfs, D. W. van Krevelen and $H$. I. Waterman on the carbonization of synthetic polymers containing aromatic units linked by methylene bridges labelled with radioactive carbon; their fate on pyrolysis could be readily followed. The course of chemical decomposition was thus related to other measurements on the polymer, for example, in a dilatometer test and a thermobalance. 
M. F. Kessler and V. Večeřiková reported a variety of physical and physicochemical measurements on cokes and related them to hardness and reactivity. J. H. Ehretsmann and R. C. Seymour had studied the gaseous products and thermochemistry of the formation and decomposition of surface oxides on charcoals. H. Guérin and M. Bastick demonstrated the importance of microporosity during gasification of coke between $800^{\circ}$ and $1,000^{\circ}$ C. C. Heuchamps, L. Bonnetain, X. Duval and M. Letort showed that the apparent energy of activation of the combustion of graphite varied with temperature and extent of combustion, and was a complex function of heterogeneity, porous structure, impurities and reaction mechanism.

It is appropriate after three successive occasions when the burden of organization has largely been borne by the Dutch State Mines to add an appreciation of the magnificent arrangements made by the scientific and administrative staff of that body. The never failing resourcefulness of $\mathrm{Mr}$. W. J. R. Berkx in all aspects is especially worthy of mention. The moving spirit behind these conferences has been the president, Prof. D. W. van Krevelen, and it must be recorded with regret that he has now resigned the presidency in view of his pending transfer from the coal to the plastics industry. To mark the occasion, tributes were paid at the end of the Conference by R. Loison, I. G. C. Dryden and M.-Th. Mackowsky on behalf of the French-, English- and Germanspeaking sections of the audience. Prof. van Krevelen's impact on coal science has occurred only within the past ten years; yet, owing to the original and prodigious output of his research teams, he has left nothing unchanged. The Conference wished him every success in his new field of endeavour.

I. G. C. Drydey

\section{CURRENT RESEARCH ON NOISE}

$I^{\mathrm{T}}$ is only within recent years that acoustic noise, once studied simply for its effect on hearing and annoyance, has proved important in physical systems. Noise has long been a source of annoyance; its effects on working efficiency have been clear when it has interfered with speech communication, less clear in causing fatigue ; and for years it has been realized that continued excessive noise can cause permanent deterioration of hearing-_boilermaker's deafness' has long been known.

These effects are still with us, in intensified form, since with the increased power of machines to-day, noise-as a by-product of this power-is increasing also. For this reason, the physical aspects of noise have come to the fore. The intense fluctuating pressures in the noise field near a jet engine can fatigue and fracture the structure of the aircraft. The forces on a missile flying through rough air may consist largely of random constituents, which need to be analysed in some detail to determine whether any frequencies are present which may damage the equipment in the missile. Such analysis is, of course, similar to that used in many other fields, such as radar; and thus acoustic noise problems find their counterparts in other studies.

These different aspects of noise were explored recently in two symposia held by the Acoustics Group of the Physical Society. The first, "Recent Studies of Noise Problems", was held at the Imperial College of Science and Technology, London, on March 24, with four papers on psycho-acoustic problems, and two on physical acoustics.

\section{Human Response to Noise}

The general effect of the first group of papers, curiously, was that work in many aspects of the subject culminated some five years ago in the United States, and little advance has been made since. This was particularly true of the account of noise and hearing loss given by Prof. W. Burns (Charing Cross Hospital Medical School). This subject is still heavily in debt to the classic report $(Z 24-X-2)$ of the American Standards Association, and little more has been learnt about safe noise-levels. However, work is continuing on the effect of age on normal hearing; this will give a better 'base-line' for the estimation of hearing damage, and is a subject on which the American report was not very satisfactory.

Procedures for evaluation of the loudness of a complex sound, however, have progressed very little in any country. This was discussed by Mr. N. Fleming and Mr. D. W. Robinson (National Physical Laboratory). The National Physical Laboratory equal-loudness contours remain unchallenged, but they are for pure tones only. The various ways of dividing and weighting the spectrum, in order to calculate loudness, do not seem very productive; while annoyance is a factor even harder to measure objectively.

Much of this difficulty may be due to the fact that pure tones have in the past been considered of major importance. But as speech, and even music, are in effect successions of transients, many noises should be considered in the same way; and a study of the aural effects of transients may be a more fruitful approach.

The point was, however, made by $\mathrm{Mr}$. Fleming that the multiplicity of criteria in American psychoacoustic studies may not be needed. He pointed out the equivalence between the 'equal-annoyance' contours for community noise, and the readings of representative noises on the $A$ weighting of the sound-level meter. The $A$ weighting is a valuable one for many approximate loudness studies; it is frequently used on the European continent in trafficnoise problems.

Further points on community reaction to noise were dealt with by Mr. H. J. Purkis (Building Research Station). The techniques for evaluating the annoyance of different types of noise, developed in America some years ago, still appear generally valid, although some alterations to suit the differing susceptibilities of English communities are needed. It is hoped to collate several case histories of noise annoyance, with the view of obtaining more applicable data.

Dr. D. E. Broadbent (Medical Research Council) spoke on the effects of noise on working efficiency. This is a field in which it is most difficult to obtain conclusive results. A pattern is, however, beginning to emerge, connected with the effect of noise in interrupting attention; noise often seems to interfere with tasks necessitating short-term memory. An overall level of $90 \mathrm{db}$. appears critical, and it is found 